\title{
Actinobacteria vs. Fusarium: Battle of the toxins
}

\author{
Laura De Mets ${ }^{1}$, Kris Audenaert², Leen De Gelder ${ }^{1}$
}

${ }^{1}$ Lab. of Environmental Biotechnology, Dept. of Biotechnology, Faculty of Bioscience Engineering, Ghent Univeristy,

2 Laboratory of Applied Mycology and Phenomics, Dept. of Crop Protection, Faculty of Bioscience Engineering, Ghent University

\section{Introduction}

Fusarium head blight is a devastating disease in small grain cereals caused by the Fusarium Head Blight species complex, of which Fusarium graminearum is the most important. Next to deteriorated quality of the grains, mycotoxins are a major health concern that challenges the global food chain. In order to control Fusarium Head Blight, an integrated crop management system is needed.

In this study, we screened a collection of Actinobacteria for their use as biocontrol agents. After an initial screening using dual culture assays, we looked into the potential of the most promising strains for application at different stages of the Fusarium Head Blight disease cycle.
Overview of screening results

$62 \%$ of 53 screened strains showed significant growth inhibition of F. graminearum $\mathrm{PH} 1(\mathrm{p}<0,05)$

S. rimosus strains caused almost complete growth inhibition and one strain showed clear growth reduction. These 3 strains were further studied.

of biological repeats was not normally distributed and showed high variation in pigmentation, sporulation and growth of the fungus, indicating an interaction between pathogen and biocontrol agent.
Screening for growth inhibition in a dual culture assay

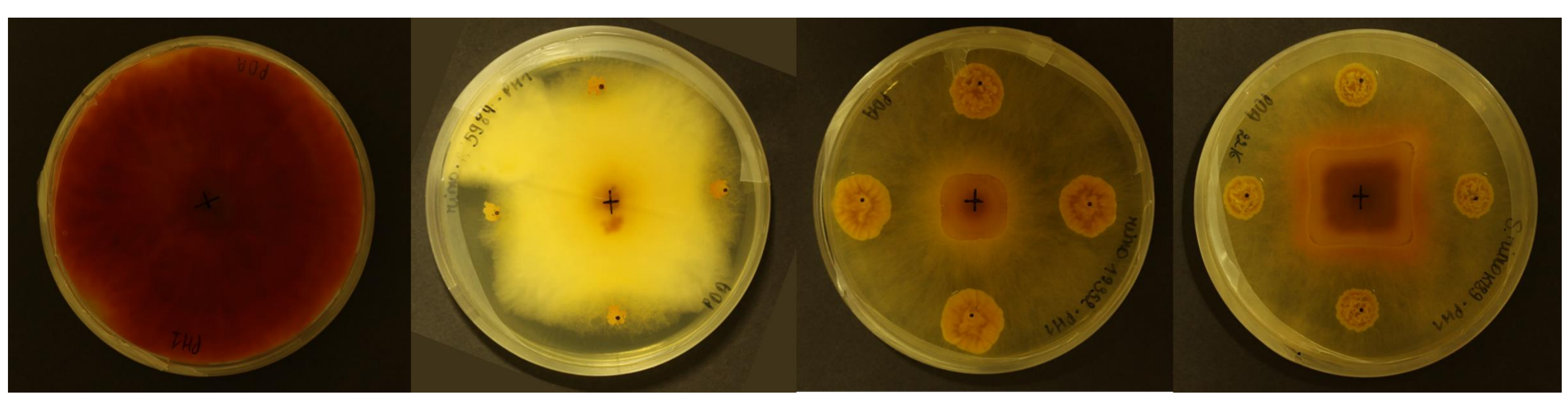

Two out of three screened $S$. rimosus strains showed high growth inhibition of $F$. graminearum $\mathrm{PH}$, while one showed clear growth reduction. When a slot was cut out off the agar plates, this inhibition diminished, indicating the plausible use of (a) diffusible component(s)

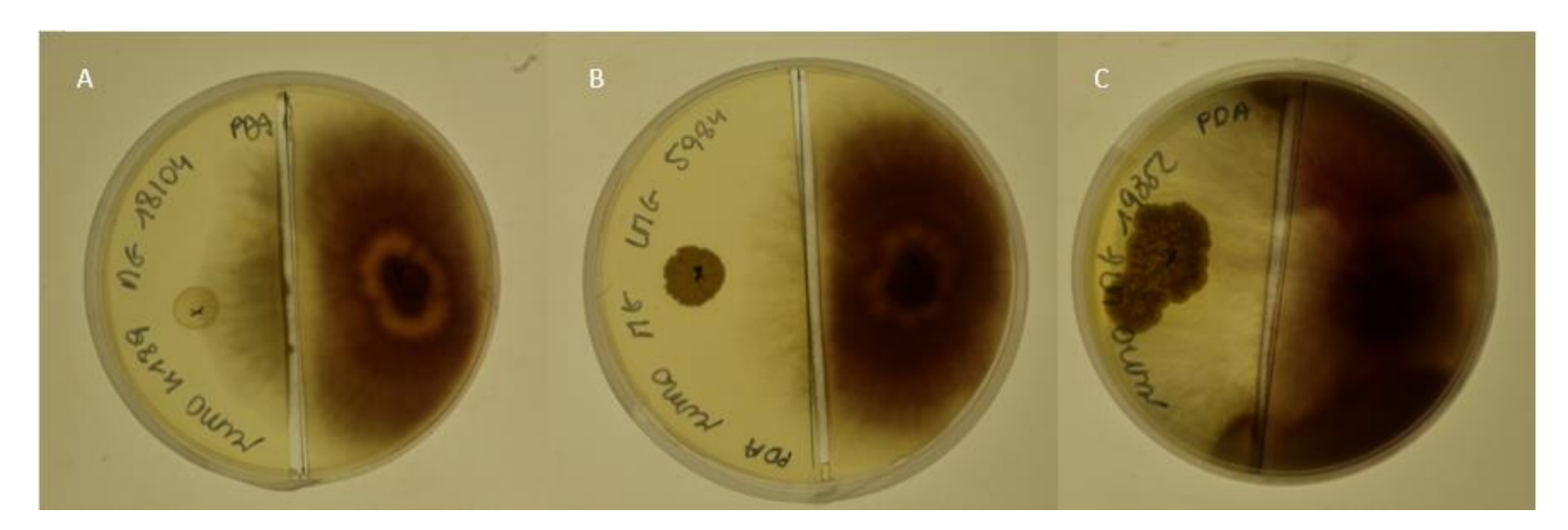

Loss of perithecia formation on wheat stubble

The application of the three $S$. rimosus strains on wheat stubble was tested.

First, the soaked and sterilized wheat stubble pieces were inoculated with $F$. graminearum. After 48h, the stubble was treated with an overnight grown and washed $S$. rimosus culture. For all three strains, we could visually see a loss of perithecia formation.
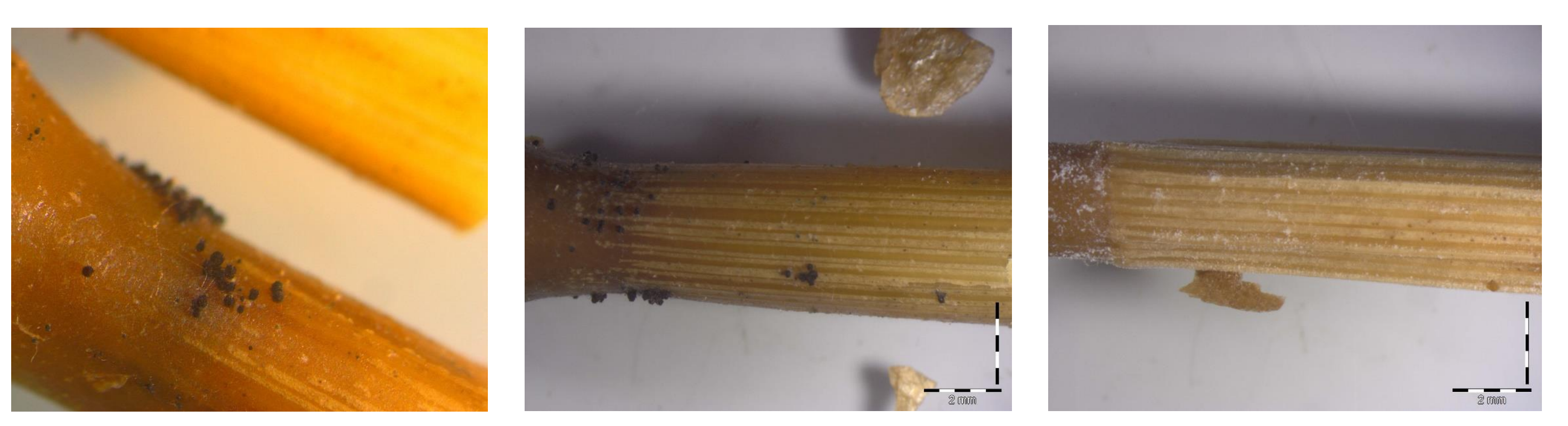

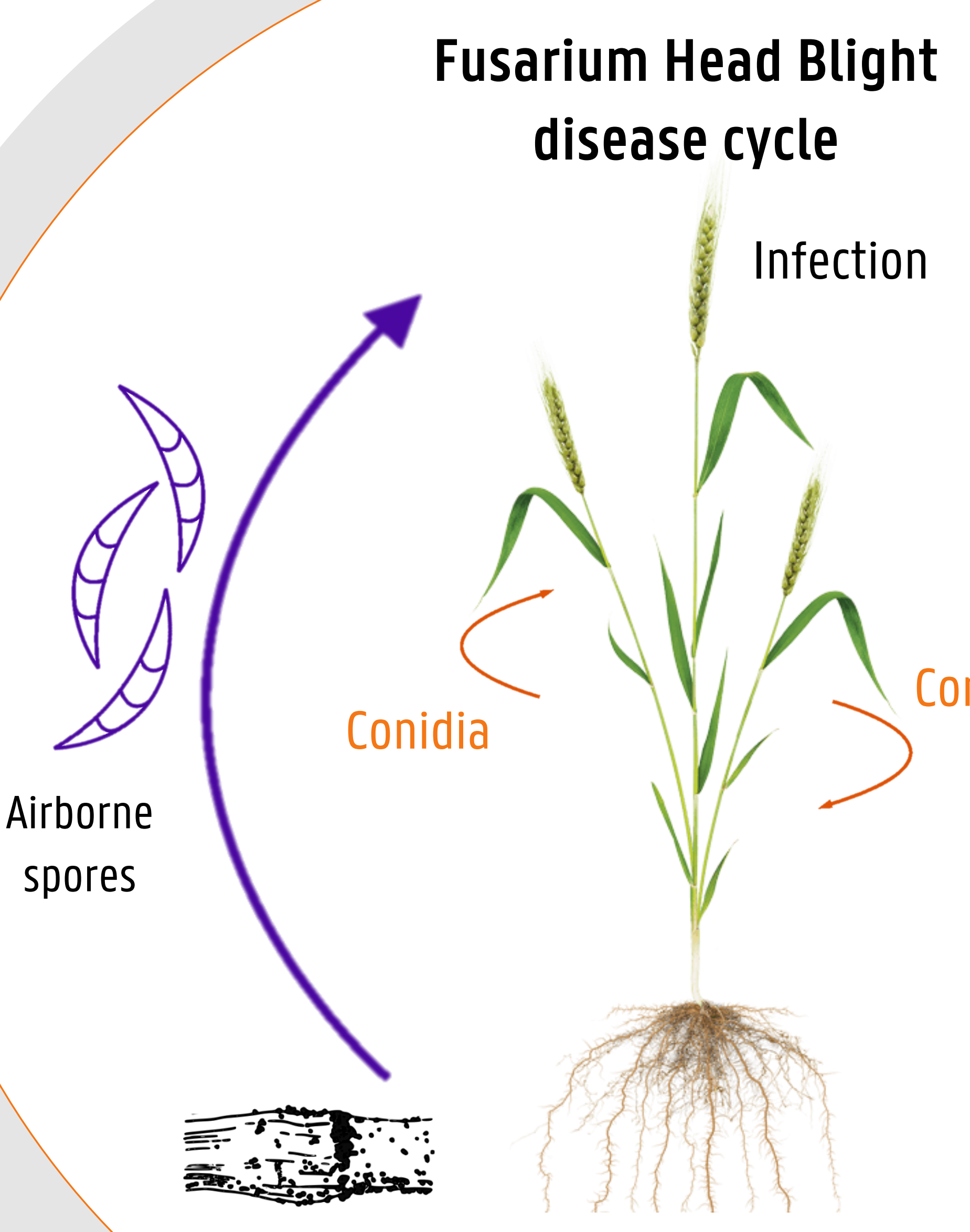

Survival on crop residues through perithecia formation
Degradation of mycotoxic virulence factors and defence mechanisms

\begin{tabular}{|ccccc}
\hline $\begin{array}{c}5 \mathrm{mg} / \mathrm{L} \text { in LB } \\
\text { After } 72 \mathrm{~h}\end{array}$ & $\begin{array}{c}\text { Degradation } \\
\text { (LC-MS/MS) }\end{array}$ & $\begin{array}{c}\text { Detoxification } \\
\text { (BLYES/BLY) }\end{array}$ & $\begin{array}{c}\text { Degradation } \\
\text { (HPLC-UV) }\end{array}$ & $\begin{array}{c}\text { Detoxification } \\
\text { (Lemna minor) }\end{array}$ \\
$\begin{array}{c}\text { S. rimosus LMG } \\
5984\end{array}$ & $100 \%$ & $98 \%$ & $0 \%$ & n.a. \\
$\begin{array}{c}\text { S. rimosus LMG } \\
\quad 19352\end{array}$ & $100 \%$ & $99 \%$ & $0 \%$ & n.a. \\
\hline S. rimosus K189 & $100 \%$ & $98 \%$ & $0 \%$ & n.a.
\end{tabular}

All three strains were able to degrade zearalenone (ZEN), a molecule produced by $F$. graminearum as part of its defence against mycoparasites. However, the strains did not degrade deoxynivalenol (DON), an important virulence factor.

This shows that the biocontrol and loss of virulence observed in the other assays, is most likely not related to degradation of DON. The strains are however able to hijack $F$. graminearum's defensive efforts.

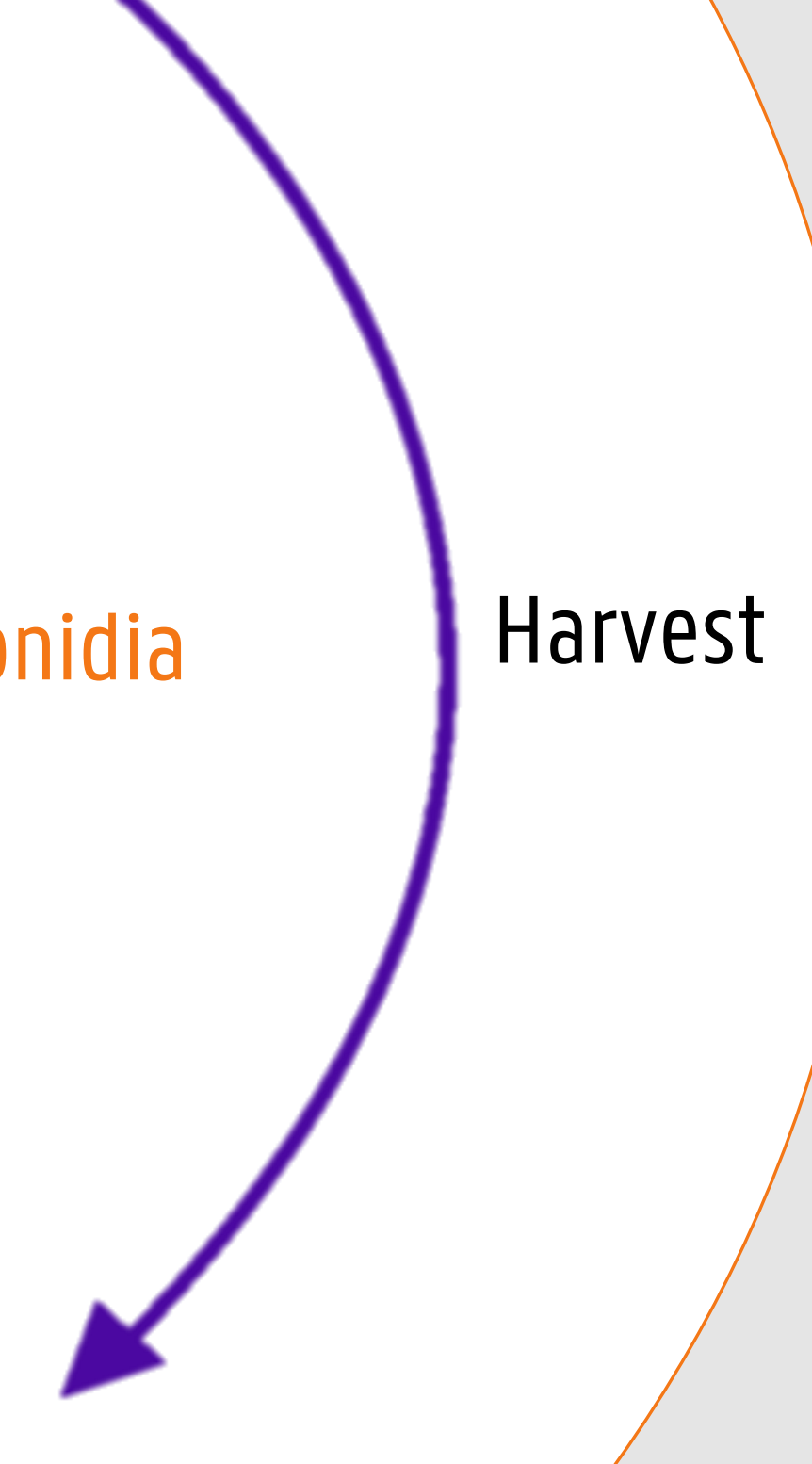

Reduced virulence and fungal

growth on detached wheat leaves

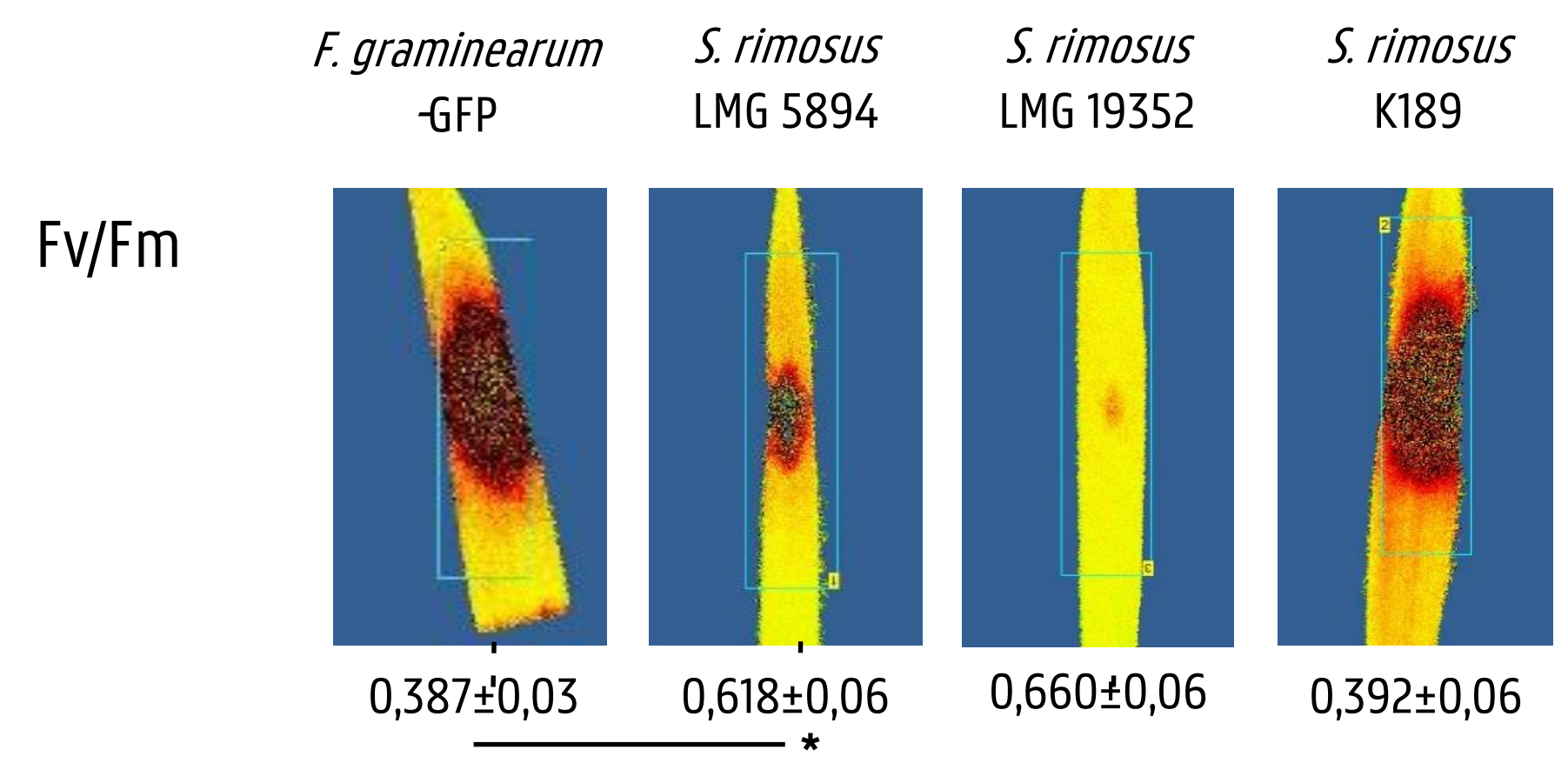

GFP

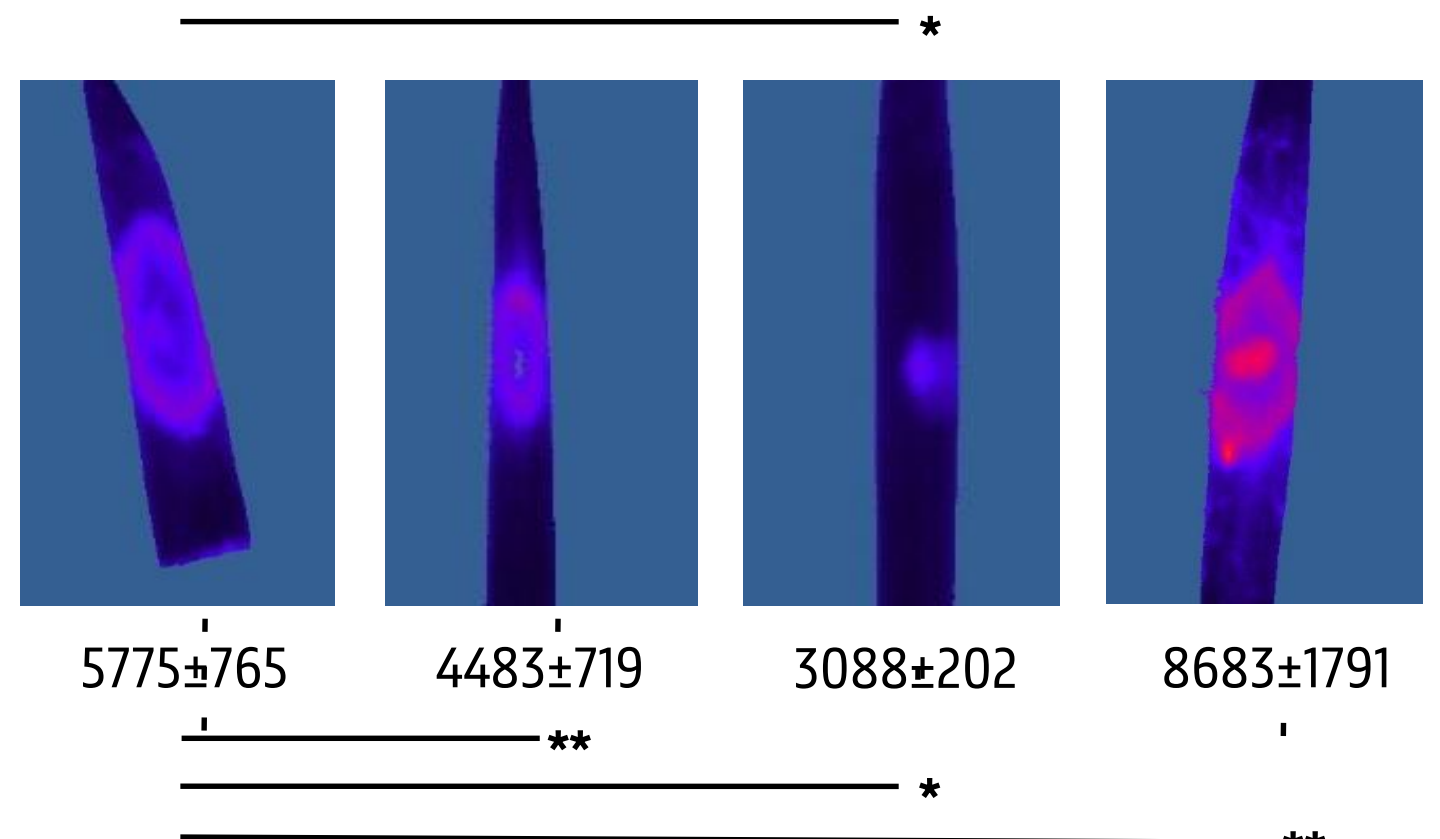

${ }^{*} p<0,001 ;{ }^{* *} p<0,05$
The effect of the three $S$. rimosus strains on $F$ graminearum virulence was tested on detached wheat leaves. A GFP-tagged F. graminearum strain was used. Virulence of infection was measured through $\mathrm{Fv} / \mathrm{Fm}$ and active fungal biomass was assessed as GFP fluorescence. All images were made with the PathoViewer phenotyper.
Take-home messages - The screened Actinobacteria were highly bioactive against F graminearum $\mathrm{PH}$, with $62 \%$ causing significant growth reduction.

- Two S. rimosus strains show high potential as biocontrol agents at different stages of the $F$. graminearum life cycle. 